\title{
Desarrollo de la competencia transversal “Aplicación Pensamiento Práctico” en la asignatura Riesgos en Instalaciones Industriales y Agroalimentarias
}

\author{
Sergio Gallardo ${ }^{a}$, Sofía Carlos ${ }^{a}$, Sebastián Martorella, José Felipe Villanuevab y Ana \\ Isabel Sánchez ${ }^{b}$ \\ àDepartamento de Ingeniería Química y Nuclear, Universitat Politècnica de València, bepartamento \\ de Estadística e Investigación Operativa, Universitat Politècnica de València.
}

\begin{abstract}
The course "Industrial facilities and food processing industries" belongs to the Master's Degree in Occupational Risk Prevention of the Universitat Politècncia de València. The course is focused on safety regulations and analyzes generic industrial facilities and food processing industries. During the last year, 18 students were enrolled in the course, with the difficulty of coming from degrees of very different knowledge areas. From a preliminary analysis of the current situation, it has been found that students do not perceive the subject as something complete but rather as a disjointed set of regulations, hindering their application to complex real problems where different regulations and facilities are involved. This problem is in the frame of the transversal skill "Application of Practical Knowledge" To address this problem, the use of the "Project" methodology is proposed. One of the most important challenge of the application of this methodology is to assess its effectiveness in terms of acquisition of a studied skill. For this purpose, some indicators have been followed over the past five years (detailed contents califications and satisfaction surveys) and the correlation between these indicators and previous academic training of students has been analyzed. We studied if there is a significant variation in the indicators, especially for students of non-technical profile, after application of this methodology.
\end{abstract}

Keywords: Transversal skill; Application of Practical Knowledge; Master's Degree in Occupational Risk Prevention; Industrial facilities and food processing industries. 
Desarrollo de la competencia transversal "Aplicación Pensamiento Práctico" en la asignatura Riesgos en Instalaciones Industriales y Agroalimentarias

\begin{abstract}
Resumen
La asignatura "Control de Riesgos en Instalaciones Industriales y Agroalimentarias”pertenece al Máster de Prevención de Riesgos Laborales de la UPV y en ella se analiza la normativa de seguridad aplicable a instalaciones de industrias genéricas y agroalimentarias. La asignatura la cursan actualmente unos 18 alumnos de perfil multidisciplinar, incluyendo formaciones previas no técnicas. Se ha detectado a partir de un análisis de la situación actual, que los alumnos no perciben la asignatura como algo integrador sino más bien como un conjunto inconexo de normativas, dificultando su aplicación a problemas reales complejos donde intervienen diferentes normativas e instalaciones. Este problema se enmarca, entre otras, en la competencia transversal de "Aplicación Pensamiento Práctico". Para atacar este problema, se propone el uso de la metodología o actividad de aprendizaje de "Proyecto". Uno de los mayores retos de la aplicación de esta metodología es evaluar su efectividad en cuanto a la adquisición de la competencia estudiada. Con este objetivo, se han estudiado algunos indicadores durante los últimos 5 años (calificaciones detalladas por contenidos y encuestas de satisfacción) y se ha analizado la correlación entre dichos indicadores y la formación académica previa de los alumnos. Se ha estudiado si existe una variación en los indicadores, sobre todo, de los estudiantes de perfil no técnico, después de la aplicación de esta metodología.
\end{abstract}

Palabras clave: Competencia transversal; Aplicación pensamiento práctico; Máster de Prevención de Riesgos;Control de Riesgos en Instalaciones Industriales y Agroalimentarias.

\title{
Introducción
}

En el marco del Espacio Europeo de Educación Superior (EEES), las competencias específicas y transversales de las diferentes asignaturas adquieren una gran importancia, ya que representan la base y la finalidad de los estudios universitarios y su armonización en Europa. En este ámbito se encuentra el Máster de Prevención de Riesgos Laborales de la Universitat Politècnica de València. En el módulo Común y en la materia "Técnicas de prevención de riesgos laborales” (cuatrimestre B), está programada la asignatura "Control de Riesgos en Instalaciones Industriales y Agroalimentarias” (CRIIA). Se trata de una asignatura de 5 créditos con una docencia de 4 horas semanales (una sesión por semana). En ella se describen las instalaciones industriales y agroalimentarias más habituales y cuál es la normativa actual aplicable en España. La asignatura tiene un marcado carácter teórico (técnico y normativo) y práctico (aplicación de la parte técnica y normativa).

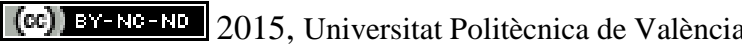


Las principales competencias específicas de la asignatura son:

- Aplicar la legislación y normativa técnica específica en materia de seguridad en el trabajo. Identificar los riesgos específicos y planificar la protección colectiva y la individual. Desarrollar planes de emergencia y autoprotección.

- Diseñar medidas preventivas para la eliminación y/o reducción de riesgos. Efectuar inspecciones de seguridad, investigación de accidentes, análisis y evaluaciones de riesgos.

- Técnicas de prevención de riesgos laborales.

- Aplicar las bases técnicas para analizar riesgos y proponer medidas correctoras en las instalaciones industriales con mayores riesgos: instalaciones eléctricas, recipientes e instalaciones a presión, instalaciones de gases combustibles o almacenamiento de productos peligrosos. En CRIIA se analizan instalaciones y normativas muy dispares: por ejemplo, equipos a presión, aire comprimido, instalaciones frigoríficas, calderas, residuos, etc. La docencia está planteada de manera que en cada sesión se analiza un determinado tipo de instalación industrial desde el punto de vista técnico y normativo.

En charlas informales con alumnos de cursos anteriores y mediante encuestas de satisfacción propias, se ha detectado una falta de percepción de la asignatura como un conjunto integrado, sino más bien como un conjunto de normativas e instalaciones poco relacionadas entre sí, y por lo tanto, perdiéndose la visión global del conjunto. Este hecho representa un inconveniente desde el punto de vista de la percepción de la utilidad de esta asignatura por parte de los alumnos. En las pruebas de evaluación realizadas hasta la fecha, se confirma que los alumnos tienen dificultades para identificar el problema y para aplicar la normativa necesaria en cada caso. En el presente curso académico, la asignatura tiene 18 alumnos matriculados. El público objetivo está compuesto por una variedad heterogénea de alumnos provenientes de una gran diversidad de titulaciones, desde ingenierías hasta medicina, lo que influye en la respuesta de las metodologías de aprendizaje utilizadas. Estas evidencias apuntan a la necesidad de trabajar, tanto metodológicamente como en su evaluación, diferentes competencias transversales. En este trabajo se analiza el desarrollo y evaluación de la competencia transversal de Aplicación del Pensamiento Práctico en esta asignatura.

La competencia transversal de Aplicación del Pensamiento Práctico se basa en facilitar la acción atendiendo a la información disponible y alcanzar los objetivos de manera eficiente. Esta competencia está relacionada con los dominios de la planificación, pensamiento creativo, resolución de problemas, toma de decisiones, etc [1]. El Pensamiento Práctico permite explotar capacidades y recursos de manera eficiente en tres niveles de dominio distintos: situaciones habituales y situaciones nuevas o complejas (de manera autónoma o colaborativa). El desarrollo de esta competencia implica trabajar diferentes aspectos tales como la identificación de objetivos, uso de la información, análisis de la información en

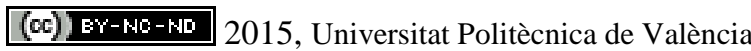

Congreso In-Red (2015) 
Desarrollo de la competencia transversal "Aplicación Pensamiento Práctico" en la asignatura Riesgos en Instalaciones Industriales y Agroalimentarias

términos de confiabilidad, entre otros [2, 3, 4] . La Tabla 1 lista los diferentes niveles de dominio y los indicadores asocidados según “Aprendizaje basado en competencias” de Aurelio Villa [1].

Tabla 1. Listado de indicadores por niveles de dominio

\begin{tabular}{|c|c|}
\hline $\begin{array}{l}\text { Nivel de } \\
\text { dominio }\end{array}$ & Indicadores \\
\hline $1^{0}$ & $\begin{array}{l}\text { 1. Identifica los objetivos concretos a lograr en las tareas que se le } \\
\text { encomiendan. } \\
\text { 2. Utiliza correctamente los elementos de información de que dispone. } \\
\text { 3. Tiene en cuenta criterios claros para evaluar la calidad de los } \\
\text { elementos de información que se le facilitan. } \\
\text { 4. Utiliza correctamente los procedimientos de procesamiento de la } \\
\text { información correspondientes a la materia. } \\
\text { 5. Traduce en decisiones o acciones concretas su análisis de la situación. }\end{array}$ \\
\hline $2^{o}$ & $\begin{array}{l}\text { 1. Establece objetivos concretos en relación con la situación que se le } \\
\text { plantea. } \\
\text { 2. Identifica los elementos de información necesarios para hacer frente } \\
\text { a la situación. } \\
\text { 3. Establece sus propios criterios para evaluar la validez de la } \\
\text { información. } \\
\text { 4. Selecciona los procedimientos adecuados para procesar la } \\
\text { información. } \\
\text { 5. Elabora un plan coherente para resolver la situación. }\end{array}$ \\
\hline $3^{\circ}$ & $\begin{array}{l}\text { 1. Acuerda objetivos concretos apropiados para la situación que se le } \\
\text { plantea. } \\
\text { 2. Acuerda el conjunto de elementos de información necesarios para } \\
\text { hacer frente a la situación. } \\
\text { 3. Acuerda criterios comunes para evaluar la validez de los elementos } \\
\text { de información. } \\
\text { 4. Acuerda los procedimientos a utilizar para procesar la información. } \\
\text { 5. Acuerda un plan coherente para resolver la situación. }\end{array}$ \\
\hline
\end{tabular}

En la asignatura CRIIA se va a trabajar con el primer nivel de dominio, ya que los indicadores se adaptan de manera adecuada al enfoque dado por los profesores que imparten docencia en dicha asignatura. Más específicamente, se va a trabajar con los indicadores 1, 2, 4 y 5 del primer nivel de dominio (Ver Tabla 1). El siguiente problema al que nos enfrentamos a la 
hora de plantear, implantar y desarrollar esta competencia en la práctica, es cómo se van a trabajar y seguir los indicadores asociados al nivel de dominio escogido. Con este objetivo, se ha determinado que el uso combinado de la metodología de aprendizaje basado en Proyectos junto con instrumento de evaluación de Portafolio puede ser óptima para el enfoque de la asignatura.

\section{Objetivos}

El trabajo tiene por finalidad la mejora de la competencia transversal "Aplicación Pensamiento Práctico" en la asignatura "Control de Riesgos en Instalaciones Industriales y Agroalimentarias” de forma que los alumnos adquieran una visión global de las instalaciones industriales y las normativas de seguridad exigibles y desarrollen la capacidad de aplicar las distintas normativas a problemas lo más reales posible de manera práctica.

Se utiliza una metodología de aprendizaje basada en Proyecto e instrumentos de evaluación de dichas competencias mediante Portafolio. En este marco los objetivos específicos del trabajo son:

- Trabajar la Competencia "Aplicación Pensamiento Práctico" en la asignatura.

- Analizar la repercusión de la metodología utilizada en la percepción de la asignatura por parte de los alumnos.

- Estudiar la posible correlación entre la visión de la asignatura por parte de los alumnos no técnicos y la metodología aplicada.

\section{Desarrollo de la innovación}

Para la puesta en práctica de esta experiencia se ha proporcionado a los alumnos información detallada (aunque deliberadamente incompleta) sobre una industria de fabricación de vidrio plano. Esta información consiste en un dossier sobre el proceso de fabricación del vidrio, la maquinaria, consumos energéticos y de materias primas y generación de residuos. Por otro lado, se ha proporcionado un plano en AutoCAD de dicha industria. En cada sesión (de 4 horas) se realiza una breve (1-1,5 horas) descripción de la normativa a estudiar, proponiendo ejercicios de entrenamiento. A continuación, por parejas, los alumnos deben aplicar la normativa estudiada al caso de la fabricación de vidrio.

Se ha promovido la formación de parejas multidisciplinares y en cualquier caso la imposibilidad de formar parejas únicamente con integrantes de perfil no técnico. En este curso, de los 18 matriculados, el 28\% son alumnos no técnicos con presencia de las siguientes titulaciones: derecho, fisioterapia, medicina y administración y dirección de empresas. El $72 \%$ restante, provienen mayoritariamente de Arquitectura Técnica (40\% del total) y un 32\%

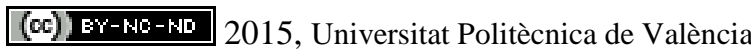


Desarrollo de la competencia transversal "Aplicación Pensamiento Práctico" en la asignatura Riesgos en Instalaciones Industriales y Agroalimentarias

de otras titulaciones técnicas. En cuanto a la procedencia de los alumnos, el $78 \%$ son españoles, mientras que el $22 \%$ restante son estudiante extranjeros.

El desarrollo de la experiencia se ha dividido en dos fases: previa a la impartición de la asignatura y durante la asignatura. Cada fase se ha dividido en una serie de actividades estableciendo un cronograma de trabajo utilizando un diagrama de Gantt y estableciendo un responsable de cada actividad.

FASE A: En esta fase, previa a la impartición de la asignatura, las actividades definidas han sido las siguientes:

1. Búsqueda bibliográfica de experiencias docentes en el desarrollo y evaluación de metodologías de aprendizaje basadas en Proyecto.

2. Búsqueda, caracterización y definición del caso a resolver durante el curso. El caso propuesto debe presentar la suficiente complejidad de forma que para resolverlo se requiera un análisis del problema con un enfoque global, su descomposición en partes más manejables y la evaluación de posibles soluciones según su viabilidad científico-técnica. En su momento se realizó un análisis de las industrias candidatas a ser utilizadas en esta experiencia. Entre ellas, se propuso una industria de fabricación de vidrio, una de fabricación cerámica y una industria alimentaria de productos lácteos. La elección de la industria objeto de análisis se realizó de acuerdo a tres criterios. Por una parte se priorizó el hecho de que los profesores tuvieran acceso a información suficientemente concreta para poder orientar los trabajos. Se estableció como segundo criterio de elección que el proceso de fabricación fuera lo más lineal posible para evitar posibles dificultades en la percepción global de la industria por parte de los alumnos. Por último, la industria debía ser susceptible de tener todas las instalaciones que se estudian en la asignatura. Finalmente, se eligío la industria de fabricación de vidrio plano. En futuros cursos se va incluir un cuarto criterio en la elección: la posibilidad de realizar visitas técnicas a una empresa real del ramo.

3. Planificar las actividades a realizar sobre el Caso referido en el apartado 2 y los métodos de seguimiento y evaluación. Esta actividad requiere un esfuerzo de programación y adaptación de contenidos a la industria elegida. Se fijó la necesidad del estudiar las siguientes instalaciones y normativas:

- Aparatos a presión: elementos de seguridad, inspección, vigilancia y mantenimiento.

- Calderas e intercambiadores.

- Equipos a presión transportables y depósitos criogénicos.

- Instalaciones gases combustibles. Almacenamiento. Instalaciones receptoras.

- Instalaciones almacenamiento productos químicos peligrosos.

- Instalaciones almacenamiento productos petrolíferos de uso propio.

(c) EY-NC-ND 2015, Universitat Politècnica de València 
- Instalaciones aire comprimido.

- Instalaciones eléctricas.

- Instalaciones frigoríficas.

- $\quad$ Residuos peligrosos.

4. Diseño de las actividades y evaluaciones. El responsable de asignatura se encarga de coordinar todos los contenidos y su planificación temporal. Las evidencias de dichas actividades se recogen en un Portafolio que al final de curso el alumno entrega a modo de dossier conteniendo cada uno de los sistemas estudiados, incluyendo la normativa aplicable, los métodos de cálculo y conclusiones. Las evidencias se completan mediante dos pruebas escritas de respuesta abierta. El Portafolio permite hacer un seguimiento del trabajo realizado y evaluarlo, así como contrastar el grado de consecución de los objetivos planteados. El Portafolio incluye una sección de planos en AutoCAD de la industria objeto de estudio, de forma que los alumnos deben plasmar en dicho plano, aspectos técnicos relativos a cada instalación, normativa, etc. Con esta medida se pretende trabajar una herramienta específica indispensable en este tipo de estudios, el diseño asistido por ordenador. En la Figura 1 se muestra una imagen del plano proporcionado a los alumnos y con el que trabajan durante toda la asignatura.

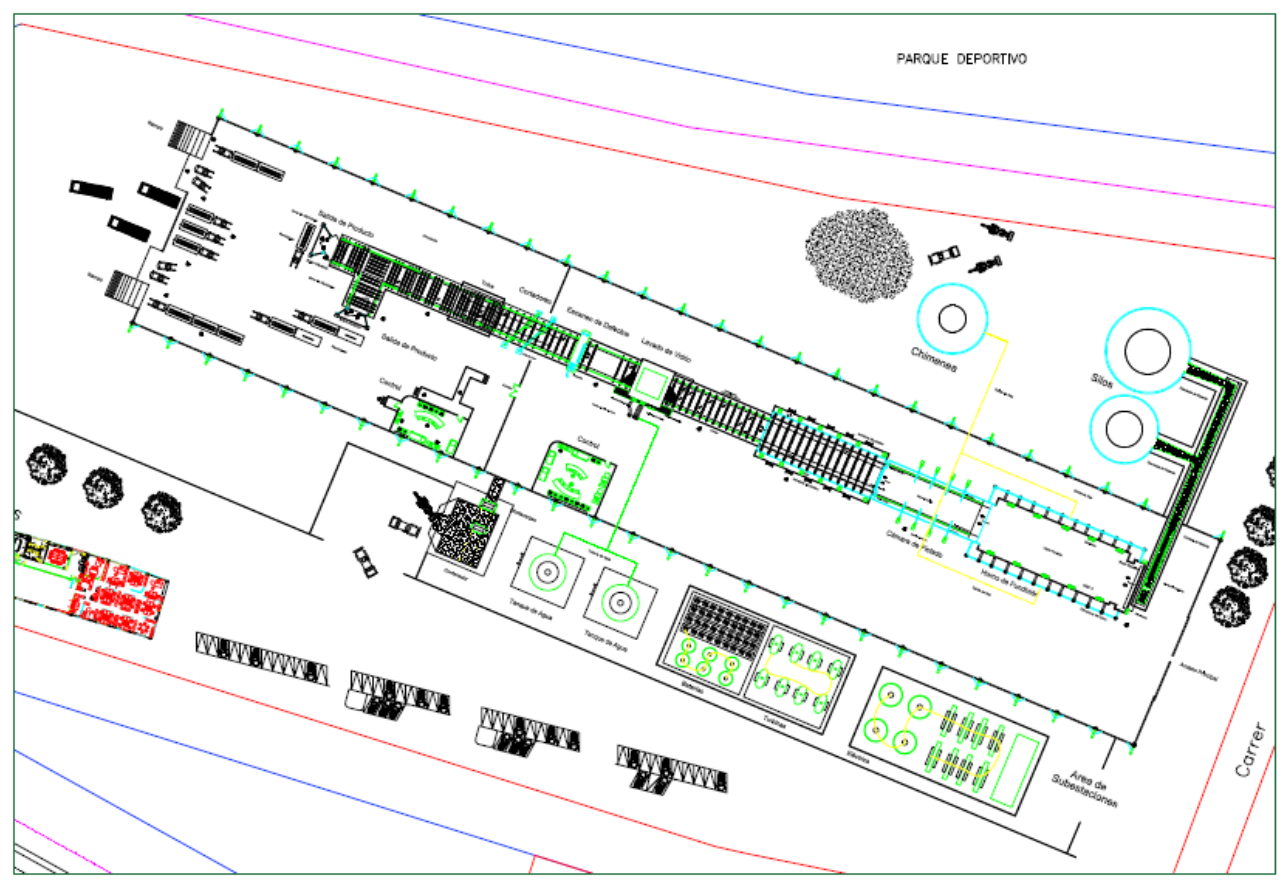

Fig. 1 Plano proporcionado a los alumnos

(cc)) EY-NC-ND 2015, Universitat Politècnica de València

Congreso In-Red (2015) 
Desarrollo de la competencia transversal "Aplicación Pensamiento Práctico" en la asignatura Riesgos en Instalaciones Industriales y Agroalimentarias

En el presente curso se ha establecido una programación temporal de entregas parciales del trabajo. Se proporciona una semana de tiempo para que los alumnos complementen y finalicen las tareas propuestas en la clase presencial. Se realiza una prueba escrita parcial con un peso total del $40 \%$ y una segunda prueba parcial (40\%) al finalizar la asignatura. El portafolio tiene un peso del 20\%. Para guardar coherencia con la metodología trabajada, las pruebas parciales se diseñan para que los alumnos respondan cuestiones de aplicación de la normativa, evitando plantear preguntas meramente teóricas.

5. Establecimiento de indicadores y herramientas para medir el grado de consecución de los objetivos del proyecto. Una de las actividades más complejas de este proyecto es intentar valorar el grado de mejora en la adquisición de las competencias de la asignatura. Para ello, es necesario cuantificar estadísticamente el impacto de su aplicación. Por este motivo, se ha realizado un estudio que incluye las evaluaciones previas a la experimentación con esta metodología y un análisis estadístico de calificaciones. Se han analizado durante los últimos cinco años, las calificaciones por contenidos de cada alumno, intentando observar una correlación entre el pefil del alumno (técnico / no técnico) y sus calificaciones en cada contenido. Otro indicador estudiado ha sido el resultado de las encuestas de satisfacción del alumnado realizados por el Instituto de Ciencias de la Educación (ICE). Todos los resultados obtenidos se detallan en la sección Resultados del presente trabajo. Por otro lado, y a partir de este curso, se realizará un cuestionario de elaboración propia para determinar el grado de satisfacción de los alumnos.

6. Coordinación y seguimiento. El responsable de las actividades planifica reuniones con una periodicidad mensual con el objetivo de distribuir y coordinar las diferentes tareas involucradas en la actividad.

FASE B: Durante a la impartición de la asignatura. Esta fase incluye las siguientes actividades:

1. Descripción de la metodología a los alumnos. En la primera sesión de la asignatura, se realiza una descripción general de la empresa incluyendo aspectos como los procesos productivos (a qué se dedica), de qué instalaciones dispone o podría disponer, cuántas personas trabajan en ella y qué riesgos laborales se pueden presentar. En este punto, se facilita a los alumnos un plano en AutoCAD de la empresa con la ubicación de la maquinaria y sus principales instalaciones. A continuación, se enumerarán las instalaciones industriales que se van a estudiar en la asignatura y su relación con el caso de estudio presentado previamente. Un aspecto que facilita en gran medida la presentación es el visionado de un vídeo de descripción general de una empresa de fabricación de vídrio, y su proceso productivo (recepción de materia prima, fundición en hornos, baños de estaño, laminado y cortado, lavado, control de calidad y expedición).

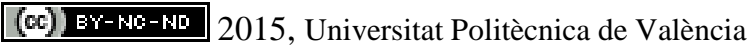




\section{Desarrollo de las actividades planificadas y diseñadas en la Fase Previa.}

Cada semana se analiza una instalación o sistema y por parejas, se aplica lo expuesto al caso de estudio. Cada sesión se inicia con una breve exposición teórica de la normativa a tratar para proceder seguidamente a la exposición del problema. Se destina aproximadamente dos horas al trabajo activo por parte de los alumnos. Se dispone de acceso a internet y PoliformaT para realizar búsquedas de información. Cada uno de los profesores de la asignatura es responsable de las tareas correspondientes a sus unidades temáticas. Por otra parte, se ha intentado potenciar el uso de la intranet de la asignatura a través de la plataforma PoliformaT. Se ha promovido la participación a través del foro de la asignatura. La temática de las sesiones se anuncia con días de antelación en la página web y se fomenta el uso del espacio compartido para el envío de las entregas parciales.

3. Seguimiento y evaluación de las actividades planificadas, diseñadas y realizadas. Se programan dos sesiones de seguimiento del estado del portafolio para fomentar una retroalimentación del alumno respecto de la consecución de las competencias de la asignatura.

4. Uso de las herramientas (definidas en la fase previa) para medir el grado de consecución de los objetivos del proyecto y obtener conclusiones al respecto. El análisis estadístico de los indicadores se ha realizado mediante el programa Statgraphics.

5. En función del análisis de los resultados se establecerán propuestas de mejora a tener en cuenta en cursos posteriores con objeto de hacerlo sostenible en el tiempo.

6. Coordinación y seguimiento. Se establece una plan de seguimiento de la aplicación de la metodología una periodicidad quincenal.

\section{Resultados}

Los resultados obtenidos se pueden caracterizar en dos tipos: cambios metodológicos en la impartición de la asignatura y resultados estadísticos relativos a calificaciones y de evaluación de la competencia.

\section{Cambios metodológicos}

- Como resultado de este trabajo, se ha modificado la estructuración y los tiempos de cada sesión, fomentando la búsqueda de información y el análisis para la toma de decisiones.

- Se ha fomentado la discusión y el debate en las sesiones prácticas. La propia definición de "Aplicación de Pensamiento Práctico" permite abrir la discusión a las simplificaciones válidas, la información no disponible, etc.

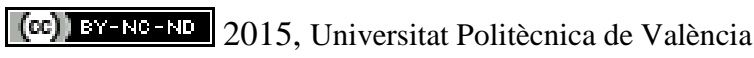

Congreso In-Red (2015) 
Desarrollo de la competencia transversal "Aplicación Pensamiento Práctico" en la asignatura Riesgos en Instalaciones Industriales y Agroalimentarias

- Se ha modificado parcialmente el sistema de evaluación de la asignatura para adaptarla a la nueva metodología. Se han introducido nuevas cuestiones, más abiertas, en las que se busca fundamentalmente la argumentación del alumno, más que un determinado resultado.

- Se ha realizado un análisis de los indicadores más adecuados para evaluar esta experiencia. Se ha intentado que estos indicadores fueran lo más objetivos posible y que no introdujeran factores ajenos a la actividad docente. Un indicador importante es el cuestionario de satisfacción de los alumnos.

- Realización de un cuestionario a los alumnos de evaluación del grado de satisfacción del alumno: a) preguntas de tipo abierto que permitan que el alumno realice propuestas de cambio y sugerencias globales para las asignaturas y b) preguntas cerradas, evaluadas en una escala 1-5, relacionadas con aspectos tales como la metodología, motivación, grado de aprendizaje y valoración general del proyecto.

- Comparación de los resultados académicos de los alumnos con los correspondientes al curso anterior.

\section{Resultados estadísticos}

Se ha llevado a cabo una recopilación de información respecto a los alumnos de los últimos cinco cursos que han cursado la asignatura. Se ha registrado información referente a los siguientes aspectos:

- $\quad$ Perfil del alumno en base a los datos facilitados a la hora de inscribirse en el Máster ( $\mathrm{T}$, técnico / N, no técnico).

- Evaluaciones parciales y finales obtenidas por los alumnos durante el desarrollo de la asignatura [0-10].

- Nota media de las evaluaciones clasificadas como de carácter práctico [0-10].

- $\quad$ Nota media de las evaluaciones clasificadas como de carácter teórico [0-10].

- $\quad$ Año de curso de los estudios (2010/2011/2012/2014).

A partir de dichos datos se han realizado diversos análisis para observar el comportamiento de las calificaciones de los alumnos con un nivel estadístico de confianza suficiente. La población de estudio está compuesta por 150 alumnos que han cursado la asignatura durante los últimos 5 años. La población consta de 31 y 119 alumnos con perfiles no técnicos y técnicos respectivamente, proporción que se ha mantenido a lo largo de cada ejercicio. En la Figura 2 se muestra el gráfico de dispersión de las medias de las evaluaciones de carácter teórico frente a las de carácter práctico, mientras que en la Figura 3 se muestran los gráficos de dispersión en función del perfil y para la evaluación teórica y práctica.

De una primera valoración se observa la existencia de posibles diferencias entre las poblaciones técnicas y no técnicas, en especial en las evaluaciones de tipo práctico, no tanto en las evaluaciones de tipo teórico. Para sustentar dicha discriminación se ha llevado sendos 
Análisis de la Varianza (ANOVA) al 95\% de confianza tomando como variables dependientes las evaluaciones medias prácticas y teóricas y como factores influyentes el carácter técnico de los estudiantes, así como el año de realización del Máster.

\section{Gráfico de Teoría vs Práctica}

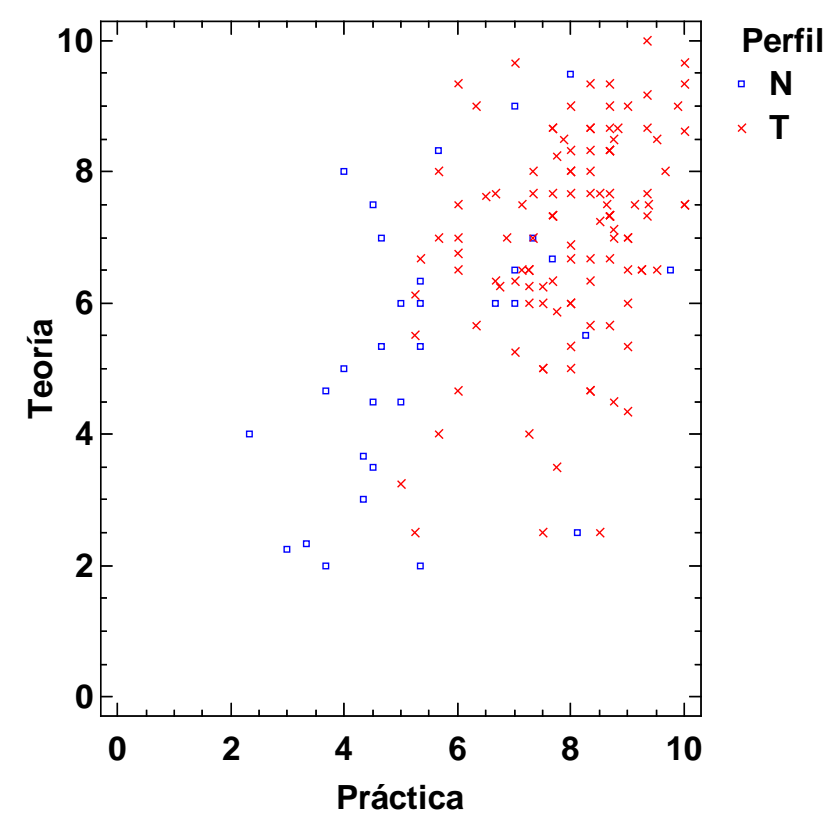

Fig. 2 Gráfico de dispersión Teoría vs Prácticas
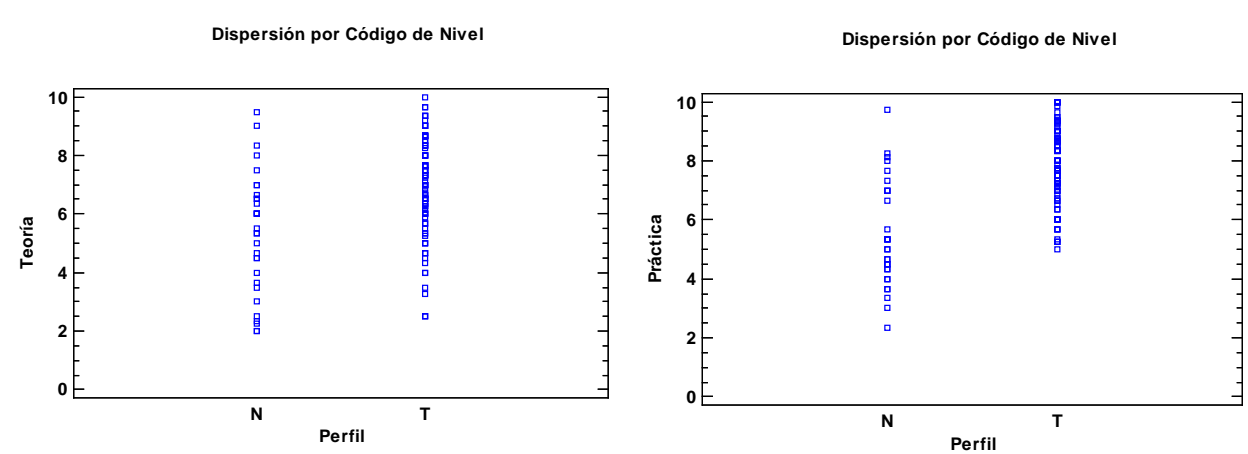

Fig. 3 Gráfico de dispersión por código de nivel para a) Teoría y b) Práctica

\section{(cc)) EY-NC-ND 2015, Universitat Politècnica de València}

Congreso In-Red (2015) 
Desarrollo de la competencia transversal "Aplicación Pensamiento Práctico" en la asignatura Riesgos en Instalaciones Industriales y Agroalimentarias

Tabla 2. Análisis de Varianza para Teoría

\begin{tabular}{lccccc}
\hline Fuente & $\begin{array}{l}\text { Suma } \\
\text { Cuadrados }\end{array}$ & Gl & $\begin{array}{l}\text { Cuadrado } \\
\text { Medio }\end{array}$ & Razón-F & Valor-P \\
\hline EFECTOS PRINCIPALES & & & & & \\
A:Año & 7,61108 & 3 & 2,53703 & 0,94 & 0,4214 \\
B:Perfil & 56,4675 & 1 & 56,4675 & 21,00 & 0,0000 \\
INTERACCIONES & & & & & \\
AB & 18,13 & 3 & 6,04332 & 2,25 & 0,0854 \\
RESIDUOS & 381,803 & 142 & 2,68876 & & \\
TOTAL (CORREGIDO) & 492,211 & 149 & & & \\
\hline
\end{tabular}

Tabla 3. Análisis de Varianza para Práctica

\begin{tabular}{lccccc}
\hline Fuente & $\begin{array}{l}\text { Suma } \\
\text { Cuadrados }\end{array}$ & Gl & $\begin{array}{l}\text { Cuadrado } \\
\text { Medio }\end{array}$ & Razón-F & Valor-P \\
\hline EFECTOS PRINCIPALES & 140,65 & 1 & 140,65 & 109,15 & 0,0000 \\
A:Perfil & 67,4559 & 3 & 22,4853 & 17,45 & 0,0000 \\
B:Año & & & & & \\
INTERACCIONES & 20,5028 & 3 & 6,83425 & 5,30 & 0,0017 \\
AB & 182,985 & 142 & 1,28863 & & \\
RESIDUOS & 415,673 & 149 & & & \\
TOTAL (CORREGIDO) & & & & & \\
\hline
\end{tabular}

A partir de este análisis se evidencia con el nivel de confianza señalado, que los resultados medios obtenidos tanto para las evaluaciones de carácter técnico como práctico se encuentran claramente afectadas por el perfil del estudiante (Figura 4), en especial en las evaluaciones de carácter más práctico, mientras que se observa que el año de realización de los estudios resulta significativo para las evaluaciones de carácter práctico, y no para las evaluaciones de carácter teórico donde no se aprecia influencia del año de realización de los estudios (Tabla 2 y Tabla 3). Todo esto confirma el supuesto inicial de que la procedencia del alumno influye sobre los resultados de esta asignatura con un fuerte componente técnico y práctico que supone una mayor dificultad para los alumnos de procedencia no técnica. 
Medias y $95,0 \%$ de Fisher LSD

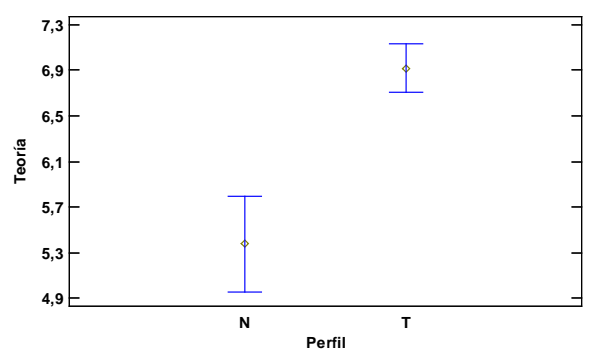

Medias y $95,0 \%$ de Fisher LSD

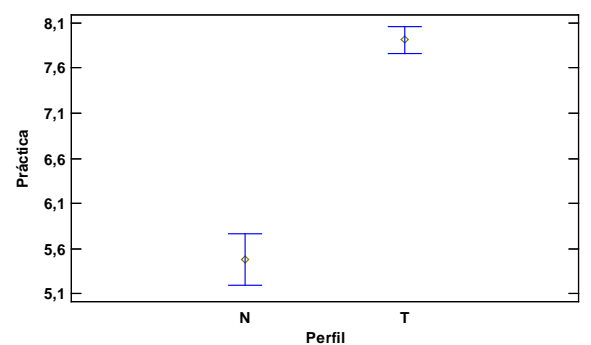

Fig. 4 Gráfico de medias y 95\% de Fisher LSD para a) Teoría y b) Práctica

\section{Resultados después de la aplicación de la metodología propuesta.}

Finalizado el curso se ha procedido a analizar los datos obtenidos respecto a las evaluaciones de carácter práctico y teórico frente al perfil técnico y no técnico de los estudiantes.

En primer lugar se ha observado una diferencia significativa entre los resultados del presente curso donde se ha incorporado la metodología frente a los cursos anteriores como se puede apreciar en la Figura 5, que muestra claramente dos poblaciones diferentes tanto en las evaluaciones teóricas como prácticas que evidencian una mejora sustancial respecto a años pasados en general para todos los alumnos.

Discriminando por el perfil de los alumnos los resultados muestran, en comparación con años anteriores, una mejora sustancial para los alumnos de perfil no técnico que mejoran sus resultados hasta igualar los de perfil técnico como se aprecia en la Figura 6.

(cc)) EY-NC-ND 2015, Universitat Politècnica de València

Congreso In-Red (2015) 
Desarrollo de la competencia transversal "Aplicación Pensamiento Práctico" en la asignatura Riesgos en Instalaciones Industriales y Agroalimentarias

Medias y $95,0 \%$ de Fisher LSD

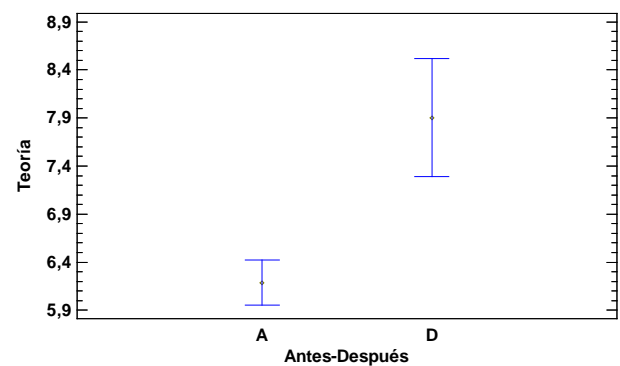

Medias y $95,0 \%$ de Fisher LSD

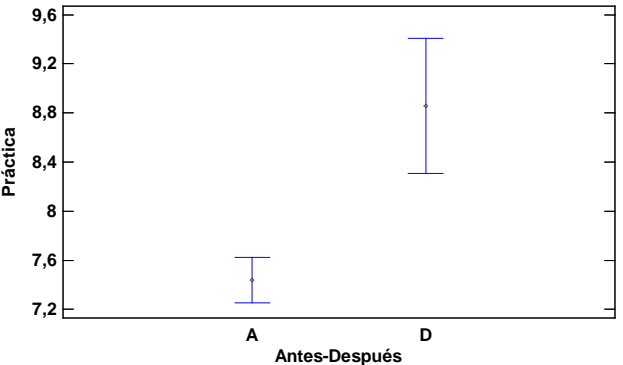

Fig. 5 Medias y 95\% Fisher LSD para a) Teoría y b) Práctica Antes y Después

Medias y $95,0 \%$ de Fisher LSD

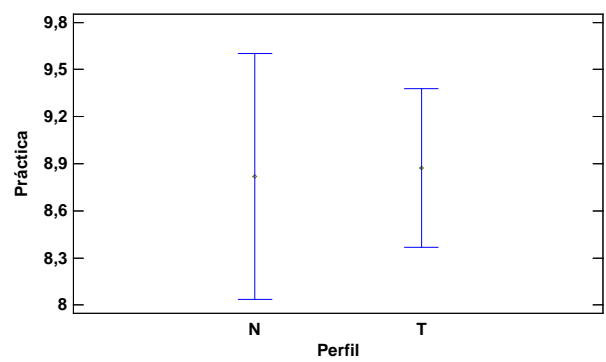

Medias y $95,0 \%$ de Fisher LSD

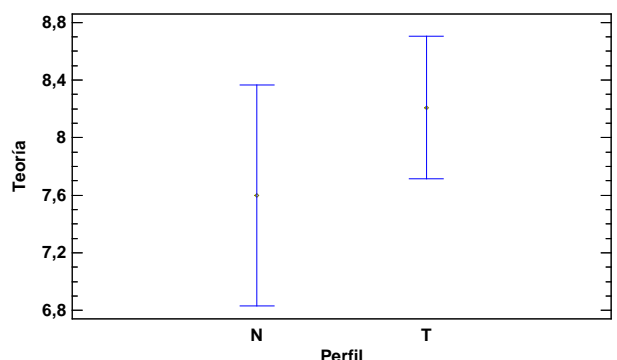

Fig. 6 Medias y 95\% Fisher LSD para a) Teoría y b) Práctica Después según Perfil

Gráfico de Interacciones

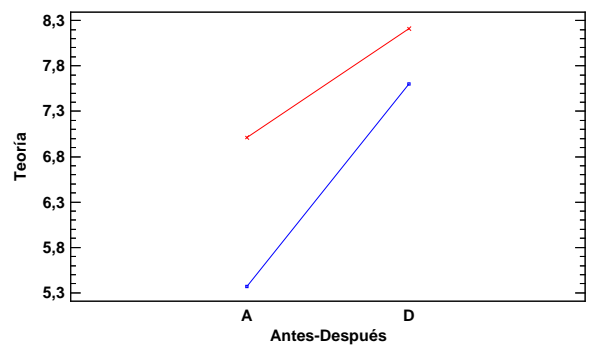

Gráfico de Interacciones

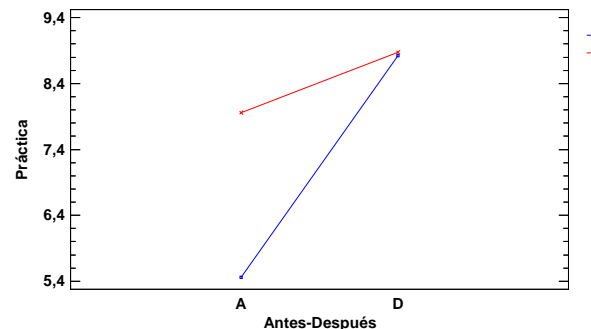

Fig. 7 Gráfico Interacción Antes-Después con Perfil para a) Teoría y b) Práctica

La mejora en la evaluación de los alumnos es general entre los dos perfiles del alumnado tanto en la pruebas teóricas como prácticas, siendo mayormente más apreciable la mejora en las pruebas más prácticas (Figura 7). 


\section{Conclusiones}

Éste ha sido el primer curso en el que se está experimentado con esta metodología, por lo que es difícil extraer conclusiones estadísticamente sólidas. Sin embargo, a nivel cualitativo sí se pueden extraer conclusiones generales. En los últimos cinco cursos, se han identificado los contenidos (normativas e instalaciones) que presentan mayor dificultad para los alumnos. Esta identificación se ha podido realizar gracias al trabajo de tratamiento de los resultados de las evaluaciones detalladas por contenidos. La estratificación de los datos en las categorías de alumnos de perfil técnico y no técnico, ha permitido encontrar diferencias significativas entre ambos perfiles respecto a cada uno de los contenidos identificados previamente, mostrando que los alumnos de perfil no técnico presentaban mayores problemas en aquellos contenidos más prácticos. Para subsanar este problema se ha trabajado preferentemente en dichos contenidos, aplicándolos a un caso real (empresa de fabricación de vidrio), de forma que implícitamente se ha involucrado a los alumnos en una situación parecida al ejercicio profesional. Además, todo ello ha permitido trabajar otras competencias tranversales muy relacionadas, como "Análisis y Resolución de problemas" e "Instrumental específica". Los resultados obtenidos con esta primera experiencia son muy satisfactorios en cuanto a la mejora de los resultados académicos, especialmente de los alumnos de perfil no técnico. Sería aventurado conceder todo el mérito de los resultados a la metodología docente empleada, ya que, los resultados pueden estar ligeramente afectados por otros factores no estudiados en este trabajo (nuevas exigencias de nota media para becas, etc). Es por ello que no se podrán extraer conclusiones más sólidas hasta dentro de unos cuantos cursos. Por otro lado, el análisis de los cuestionarios de satisfacción de elaboración propia, permite concluir que esta metodología ha representado un fuerte incremento de trabajo por parte de los alumnos y éste en el punto en el que los alumnos han sido más críticos con nosotros. Sin embargo, el resultado del cuestionario también refleja que les ha parecido una mejora importante en el enfoque de la asignatura, el hecho de trabajar sobre un proyecto determinado. En los próximos cursos se ahondará en la aplicación de la competencia transversal "Aplicación pensamiento práctico", trabajando la rúbrica de evaluación de la competencia y analizando de manera estadísticamente rigurosa (con mayor muestra) la efectividad de la metodología.

\section{Referencias}

[1] VILLA, A. y POBLETE, M. Aprendizaje basado en compentencias. Una propuesta para la evaluación de las competencias genéricas.

[2] “Problem-Based Learning”, McMaster University, http://www.chemeng.mcmaster.ca/pbl/pbl.htm, 2001.

[3] BENIT, A., CRUZ, A., “Nuevas Claves Para La Docencia Universitaria En El Espacio Europea De Educación Superior”. Madrid: Narcea S.A., 2005.

[4] GIMENO, J., Educar por competencias, ¿qué hay de nuevo? Ediciones Morata, S. L. 2008.

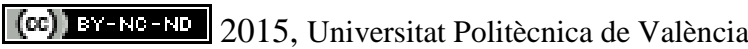

